\title{
CPInformer for Efficient and Robust Compound-Protein Interaction Prediction
}

\author{
Yang Hua, Xiaoning Song*, Member, IEEE, Zhenhua Feng*, Member, IEEE, Xiao-Jun Wu, Member, IEEE, \\ Josef Kittler, Life Member, IEEE, Dong-Jun Yu, Member, IEEE
}

\begin{abstract}
Recently, deep learning has become the mainstream methodology for Compound-Protein Interaction (CPI) prediction. However, the existing compound-protein feature extraction methods have some issues that limit their performance. First, graph networks are widely used for structural compound feature extraction, but the chemical properties of a compound depend on functional groups rather than graphic structure. Besides, the existing methods lack capabilities in extracting rich and discriminative protein features. Last, the compound-protein features are usually simply combined for CPI prediction, without considering information redundancy and effective feature mining.

To address the above issues, we propose a novel CPInformer method. Specifically, we extract heterogeneous compound features, including structural graph features and functional class fingerprints, to reduce prediction errors caused by similar structural compounds. Then, we combine local and global features using dense connections to obtain multi-scale protein features. Last, we apply ProbSparse self-attention to protein features, under the guidance of compound features, to eliminate information redundancy, and to improve the accuracy of CPInformer. More importantly, the proposed method identifies the activated local regions that link a CPI, providing a good visualisation for the CPI state. The results obtained on five benchmarks demonstrate the merits and superiority of CPInformer over the state-of-the-art approaches.
\end{abstract}

Index Terms-Compound-Protein Interaction Prediction, Drug Discovery, Compound Feature Fusion, Multi-Scale Protein Feature Extraction, ProbSparse Self-Attention.

\section{INTRODUCTION}

C OMPOUND-PROTEIN Interaction (CPI) prediction is crucial for early drug screening. Traditional experimental screening methods usually take 2-3 years, which is expensive and time-consuming. In contrast, computer-assisted virtual drug screening is much more efficient, offering significant advantages over the traditional experimental screening approaches. The classical CPI prediction methods include structure-based and ligand-based models [1], [2], [3]. These methods have gained some success in drug discovery but do not perform well in many tasks involving datasets with numerous or unknown proteins because

* Corresponding authors.

Yang Hua, Xiaoning Song and Xiao-Jun Wu are with the School of Artificial Intelligence and Computer Science, Jiangnan University, Wuxi 214122, China. (email: 7211905018@stu.jiangnan.edu.cn, x.song@jiangnan.edu.cn, wu_xiaojun@jiangnan.edu.cn)

Zhenhua Feng and Josef Kittler are with the School of Computer Science and Electronic Engineering, University of Surrey, Guildford, GU2 7XH, UK. (e-mail: \{z.feng; j.kittler\}@ surrey.ac.uk)

Dong-Jun Yu is with the School of Computer Science and Engineering, Nanjing University of Science and Technology, Nanjing, 210094, China. (email: njyudj@njust.edu.cn) most proteins lack 3D structural information [3]. Therefore, a novel method called chemogenomics was introduced to predict CPIs without reference to their 3D protein structures [3], [4]. Since then, various machine learning algorithms based on molecular information and protein sequences have been widely studied and showed to achieve promising results [3], [5], [6], [7], [8], [9], [10], [11], [12]. More recently, deep learning has become the mainstream methodology in this subject area, which has been proven to be very effective for high-performance CPI prediction [13], [14], [13], [15].

In general, a CPI predictor has two main components: feature extraction, and the compound-protein interaction prediction. The feature extraction module first extracts protein and compound features. Then the prediction module, usually in the form of a binary classifier, is used to predict the interaction between a compound and a protein using the extracted features. In many classical CPI prediction models, these two components are performed in a two-stage manner, using hand-crafted features and shallow classifiers [16], [17], [18], [13], [19]. However, such a method may not generalise well to largescale datasets. In recent years, deep learning models have been widely studied for CPI prediction. For example, Lee et al. [20] proposed DeepConvDTI that applied a deep Convolution Neural Network (CNN) to raw protein sequences and molecular fingerprints [21] for both feature extraction and CPI prediction, delivering an excellent performance on a largescale dataset. In another example, Öztürk et al. [22] used Ligand Max Common Structures (LMCS) and Protein Motifs and Domains (PDM) to enrich the ligand and protein features for performance boosting. Although this method improves the performance in the drug-target binding affinity, it requires prior knowledge, which makes it hard to apply to CPI prediction that contains complex data types.

In all these algorithms, we argue that the extraction of rich and discriminative features from proteins and compounds is the key to the success of a high-performance CPI predictor. In the existing literature, a popular and effective compound feature extraction method is to use graph networks [23]. A graph network extracts molecular features using an undirected graph that represents the structural information of a compound. For instance, Tsubaki et al. [24] and Nguyen et al. [25] used Graph Neural Network (GNN) and Graph Convolutional Network (GCN), respectively, to extract compound features, represented by the Simplified Molecular-Input Line-Entry System (SMILES). These two methods achieved promising results in various benchmarking datasets, demonstrating the capability of graph networks in compound feature extraction. 


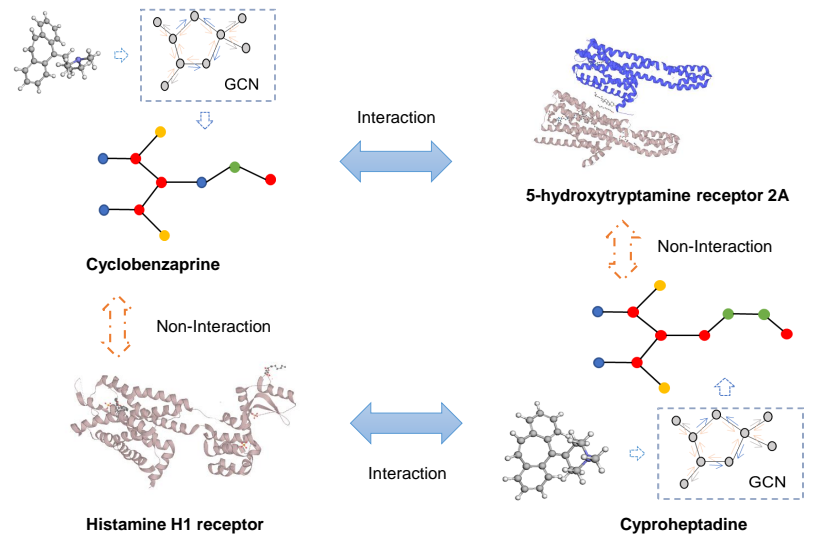

Fig. 1. Cyproheptadine and Cyclobenzaprine are two molecules with a similar planar structure. The extracted Graph Convolutional Network (GCN) features are very similar. Yet these two molecules interact with proteins very differently. This is a common issue in CPI prediction, which may lead to inaccurate prediction results when using GCN features.

Despite the success of graph networks in extracting compound features, our preliminary studies have established that they are not sensitive enough to distinguish structurally identical drug molecules. Many molecules with similar structures often have various autogenic properties. For example, Cyclobenzaprine and Cyproheptadine have analogues structures but distinct chemical properties [21]. As shown in Fig. 1, they can be regarded as the same kind of molecule when using GCN representations. However, they interact with different proteins very differently. Cyclobenzaprine interacts with the 5-hydroxytryptamine receptor $2 \mathrm{~A}$. It is a centrally acting muscle relaxant used to treat acute skeletal muscle spasm caused by musculoskeletal injury. It does not interact with the Histamine H1 receptor [26], [21]. In contrast, Cyproheptadine interacts with the Histamine $\mathrm{H} 1$ receptor but not with the 5hydroxytryptamine receptor $2 \mathrm{~A}$. It is used to relieve itching, stimulate appetite, as an anti-allergic compound, and to treat dumping syndrome after gastrectomy. With GCN features, these complex relationships may lead to instability of network training and subsequently inaccurate CPI predictions. Moreover, it is difficult to explain the interaction between compounds and proteins by biological theories, when using GCN-based molecular features. This deficiency is detrimental to drug discovery as the aim of a CPI prediction model is to explain why a compound interacts with a protein. A good CPI predictor should not only provide a high prediction accuracy but also identify the functional group characteristics of the compound molecule, improving the interpretability of the model.

In addition to the above issues, it has also been noticed that previous deep-learning-based CPI predictors often focus on predicting the type of compound, or protein, rather than predicting the CPI interactions [3]. To address this issue, Chen et al. proposed two new benchmarking datasets, GPCR and Kinese, using the label reversal protocol. In this protocol, if a ligand appears in a positive/negative pair of samples in the training set, it only appears in the opposite (negative/positive) pair of samples in the test set. These two datasets can effectively verify whether a model predicts the interaction between a compound and protein. Chen et al. also investigated a Transformer network [27], which has been widely studied in natural language processing and recently in other tasks, and proposed the TransformerCPI model that achieved state-ofthe-art performance on several benchmarks, including Human, C.elegans [28], [24], and BindingDB [29], [30]. However, the stacking of excessive self-attention mechanism in TransformerCPI increased the model parameters dramatically, and consequently reduced the efficiency of the model in both, the training and test stages.

To mitigate the above issues, we propose a novel CPI prediction model, namely CPInformer, by using three feature enhancement methods. First, we fuse the Functional Class FingerPrints (FCFPs) [31] with the structural GCN features [23] as the final representation of a compound. FCFPs contain chiral information. Each element in a fingerprint represents a specific chemical substructure of a compound, which can easily be used to explain the properties of a molecule. FCFPs features play a complementary role to the structural molecule features extracted by GCN. The fused features provide a more informative input for CPI prediction, as a compound will have complex heterogeneous features extracted from both graph structures and functional groups. Second, we improve the method for extracting features from protein sequences. Due to the complexity of a protein sequence, both the global and local features are crucial for the CPI prediction. Accordingly, we fuse multi-scale protein features extracted from different network layers with different receptive fields using dense connections [32]. Third, we base our CPInformer method on the probabilistic sparse selfattention module that was proposed for long sequence timeseries forecasting in the Informer model [33]. Previous CPI prediction models simply use the common attention modules in the existing machine learning literature. However, these methods do not question the applicability of these attention models to compound and protein data types. Different from self-attention that has been used in the existing CPI prediction methods [3], the proposed probabilistic sparse self-attention selects the area with the highest response as features for the next layer, effectively reducing information redundancy. More importantly, the probabilistic sparse self-attention module is able to identify the extent of the binding site, and determine whether it belongs to vital structural domains [34], which is essential for drug discovery research. The proposed method is also able to determine whether the compound interacts with the protein and in which region of the amino acid sequence the interaction occurs. To the best of our knowledge, this is the first work that investigates probabilistic sparse self-attention in CPI prediction.

We evaluated the proposed CPInformer method on five datasets and achieved superior performance, compared with the state-of-the-art methods. Although the use of a black-box deep learning model makes it difficult to analyse an interaction between a compound and protein from the biological point of view, we resolve this problem indirectly by using the attention mechanism [33]. We visualise the proteins and compounds based on the attention weights, rendering the protein domains 


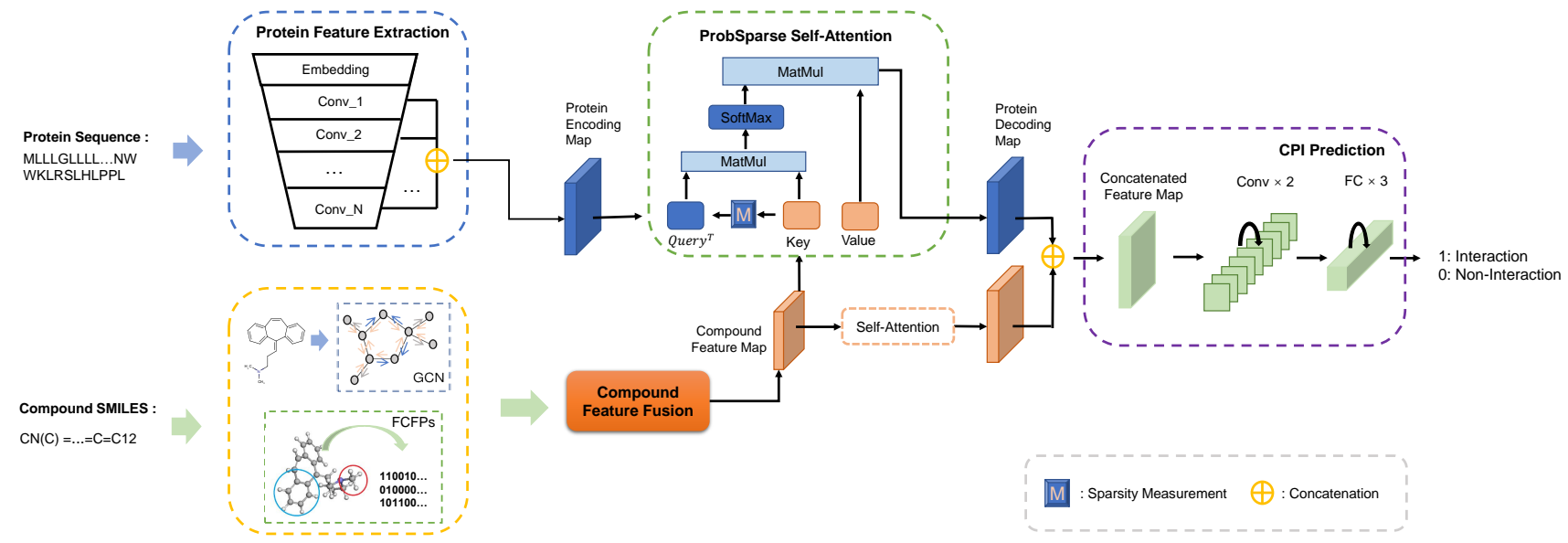

Fig. 2. The overall pipeline of CPInformer that has four main modules. The encoding module with dense connections is used for multi-scale protein feature extraction. The compound feature fusion module fuses different compound features, including FCFPs and the GCN features, for a rich feature representation. The attention module applies probabilistic sparse self-attention to protein features by using the compound features. Last, the prediction module applies a binary classifier to the concatenated compound-protein features for CPI prediction.

with a high probability of interaction, and the functional groups of compounds, to help explain why a compoundprotein interaction occurs [35].

Overall, the main contributions of our CPInformer model include:

- An Informer-based CPI prediction model. To the best of our knowledge, this is the first time that the Informer [33] model has been used for CPI Prediction.

- A feature fusion method used for rich compound feature extraction. We fuse the fingerprints and GCN features of a compound as a final representation. The aim is to reduce the inaccuracy of CPI predictions caused by the molecules with similar structures.

- A multi-scale protein feature extraction method that fuses both local and global information relating to the proteins. We apply dense connections to different network layers to achieve this goal.

- An effective attention module for compound and protein feature fusion. The proposed method significantly reduces the information redundancy, and mines discriminative compound-protein features for the subsequent prediction task.

- The visualisation of the compound-protein interactions. The proposed method is able to visualise the local regions of proteins and compounds based on the attention weights for analysing compound-protein interactions.

The rest of this paper is organised as follows. We first introduce the proposed CPInformer method in Section II. Then we report the experimental results obtained on five benchmarks in Section III. Last, the conclusion is drawn in Section IV.

\section{The Proposed CPInformer Method}

As shown in Fig. 2, the proposed CPInformer method has three innovative elements: fusion of the features of compound molecules, multi-scale protein feature extraction, and a ProbSparse self-attention mechanism. Additionally, the prediction head of the model is composed of two convolutional and three fully connected layers for binary classification. In the rest of this section, we introduce these components in more details.

\section{A. Fusion of Compound Molecules Features}

We extract compound features as shown in Fig. 3. First, we convert the SMILES formula of a compound into the feature matrix and adjacency matrix by the RDKit toolbox [36]. Then we extract the structure features of the compound using GCN, a model applying special convolution operations to a nonEuclidean diagram [37]. GCN expresses molecules through its graph structure:

$$
\begin{gathered}
\mathbf{H}^{(i)}=\operatorname{Relu}\left(\widetilde{\mathbf{D}}^{-\frac{1}{2}} \widetilde{\mathbf{A}} \widetilde{\mathbf{D}}^{-\frac{1}{2}} \mathbf{H}^{(i-1)} \mathbf{W}^{(i-1)}\right), \\
\mathbf{A}=\mathbf{A}+\mathbf{I},
\end{gathered}
$$

where $\mathbf{H}^{(i)}$ is the feature output by the $i$ th layer, $\mathbf{D}$ is the degree matrix, $\mathbf{H}^{(i-1)}$ is the feature output by the $i-1$ th layer, $\mathbf{A}$ is the adjacency matrix, $\mathbf{I}$ is the degree matrix of the compound, $\mathbf{W}^{(i-1)}$ is the weight matrix (parameters) of the $i$ th layer, and Relu() is the ReLu non-linear activation function. Subsequently, we use RDKit to obtain the Functional Class FingerPrints (FCFPs) of molecules. The functional group often determines the chemical properties of a compound [38], thus FCFPs can effectively represent its chemical features. In contrast, GCN is able to extract the structural features of the molecules, but it cannot capture the chemical properties of compound molecules. In consequence, it is unable to provide any useful information for the professional drug researchers to understand the reason behind the interaction between a compound and a protein. As GCN is also unable to distinguish compound molecules with similar planar structures, it is crucial to investigate the fusion of FCFPs and GCN features for rich and discriminative compound feature extraction.

As depicted in Fig. 3, we transform the structural GCN features and FCFPs by feed-forward neural networks and then 

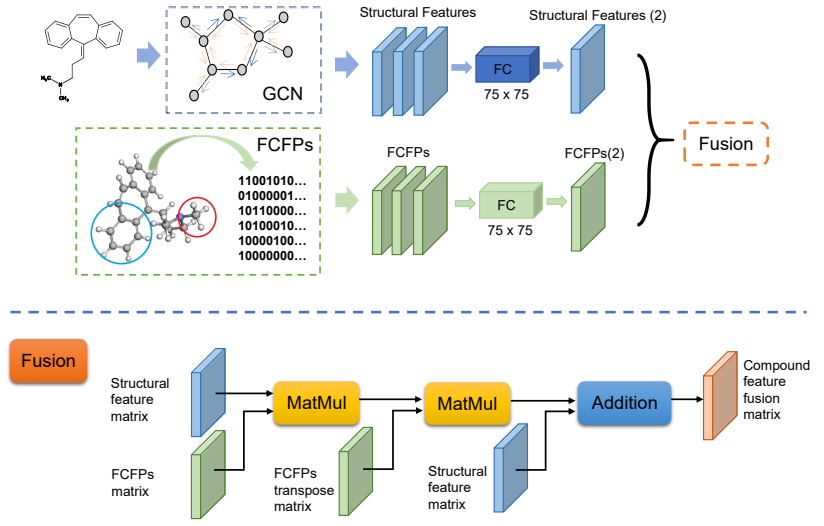

Fig. 3. We use three fully connected layers to extract the features of FCFPs and make their width the same as the width of the compound GCN feature map. This is beneficial for feature fusion. In the fusion block, 'MatMul' is the element-wise multiplication operation and 'Addition' is the element-wise addition operation.

multiply the structural feature matrix with the FCFPs transpose matrix and FCFPs matrix successively. This method is similar to self-attention, but the main aim is to fuse two different types of features effectively, instead of performing attention across the elements in the extracted features. We do not use softmax and normalisation in our compound feature fusion module because we hope the model can smoothly transfer the gradient information to the previous two modules in back-propagation. To promote the convergence and network optimisation, we add the residual block as follows:

$$
\mathbf{F}_{c}=\mathbf{F}_{g}+d_{\text {out }}\left(\left(t\left(\mathbf{F}_{g}\right) * t\left(\mathbf{F}_{p}\right)^{T}\right) * \mathbf{F}_{p}\right),
$$

where $\mathbf{F}_{c} \in \mathbb{R}^{n \times 75}$ is the fused compound feature, $n$ is the number of atoms in the compound, $\mathbf{F}_{g} \in \mathbb{R}^{n \times 75}$ is the molecular feature, $\mathbf{F}_{p} \in \mathbb{R}^{1 \times 75}$ is the FCFPs feature, and $t()$ is the FC transition function. Note that RDKit generates the initial functional group fingerprint $\mathbf{F}_{o} \in \mathbb{R}^{1 \times 1024}$ and we use three fully connected layers (with the parameter sizes of $1024 \times 512,512 \times 256$ and $256 \times 75)$ to reduce the dimensionality of the fingerprint to $\mathbf{F}_{p} \in \mathbb{R}^{1 \times 75}$. We also use the dropout layer, $d_{\text {out }}$, to prevent network overfitting.

According to our ablation study in Section III-B, the proposed compound feature fusion method improves the performance significantly. To demonstrate the advantages of the feature fusion method, we highlight the difference between the attention weights produced by GCN and the feature fusion on the adjacency matrix in Appendix A. We randomly select 24 samples from two datasets for the visualisation and show the structural perspective views of the related compounds and protein sequences.

\section{B. Multi-Scale Protein Feature Extraction}

For CPI prediction, the extraction of rich and discriminative protein features is as important as that for compound features. Protein sequences often vary in length. Both, complex macromolecular proteins and short small molecule peptide chains are very important for reliable and robust CPI prediction.
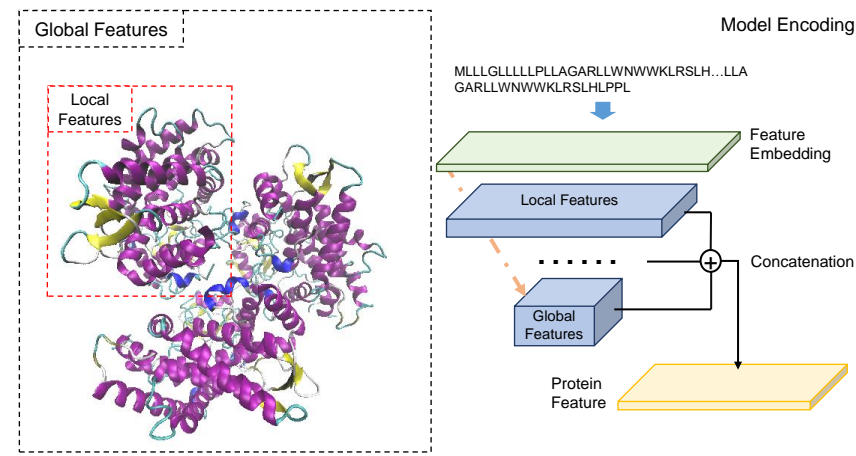

Fig. 4. Protein is a complex macro-molecular structure, and the local and overall characteristics of each region are of great significance to the judgement of CPI interactions. We fuse the features obtained by each layer densely for multi-scale protein feature extraction.

Finding an effective mapping of different protein sequences into the same feature space is very challenging because we have to capture both their local and global features [39]. To this end, we use dense connections to encode the protein data by extracting and fusing its multi-scale features across deep CNN layers, as shown in Fig. 4.

A protein sequence is usually an ordered arrangement of 20 amino acids that are common to the primary structure [40]. We follow the previous method and embed a protein sequence with three amino acid symbols to obtain its initial characteristic matrix [24]. The protein sequence in each dataset used in this paper is composed of 22 types of amino acids. If we regard the sequence as a text and denote three amino acid fragments as a word, there will be $10648\left(22^{3}\right)$ different words in the resulting vocabulary. The information described by the text may be far richer than the meaning of the protein sequence itself. In this direct case, the extracted protein features will be confusing and too sparse. To deal with this issue, we follow Che et al. [41] and divide 22 amino acids into 6 types according to their biochemical characteristics: $A=\{H, R, K\}$, $B=\{D, E, N, Q\}, C=\{C, X\}, D=\{S, T, P, A, G, U\}$, $E=\{M, I, L, V\}$ and $F=\{F, Y, W\}$. In this way, the sequence 'MSPLNQSAEGLPQEASNRSLN' is converted to 'EDDEBBDDBDEDBBDDBADEB.' The number of words is compressed to $216\left(6^{3}\right)$, reducing the protein feature redundancy significantly.

The dimension of the extracted protein feature matrix is $l \times$ 75 , where $l$ is the length of a sequence and 75 is the dimension of the word mapping space. As shown in Fig. 4, we apply multi-level convolution to the original protein feature matrix, producing multi-scale feature maps with different receptive fields. Among them, the receptive field of the first-level feature map is $k$ (the size of the convolution kernel), and the receptive field of the $n$-th layer element is $n * k-1$. The receptive field of the network for a protein sequence becomes wider as the network goes deeper.

With a sufficient number of convolution layers, the network is able to capture the global features of a protein very well. However, the feature map obtained by a deep network layer ignores local protein features. To resolve this problem, we use dense connections and concatenate the feature maps layer by 


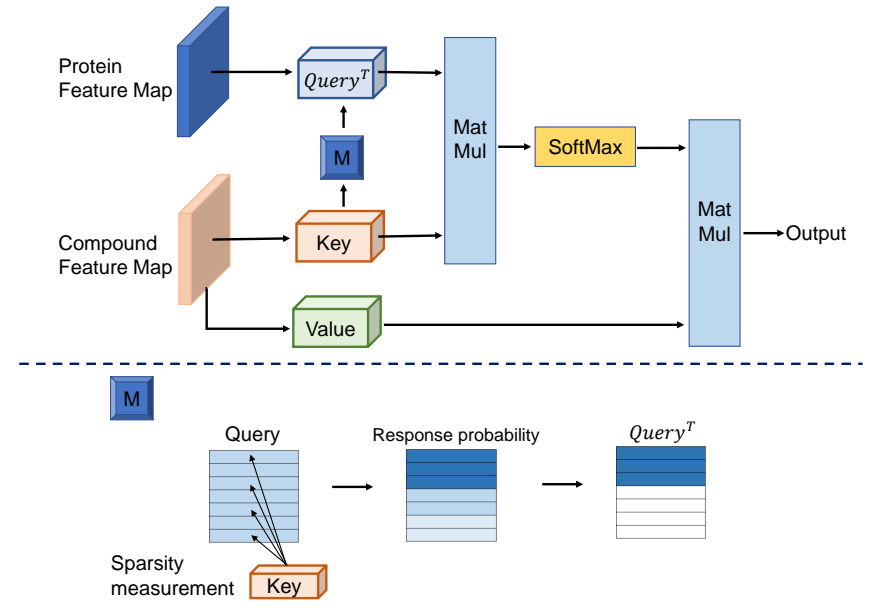

Fig. 5. Overall, the ProbSparse self-attention mechanism is similar to the common self-attention mechanism. However, The ProbSparse self-attention mechanism sorts the vectors on the Query according to the probability sparsity between the Query matrix and the Key matrix. We retain the top-u vectors as the new Query matrix and reset the rest of the vectors to zero. The new Query matrix and the Key matrix generate the attention weight matrix.

layer, in which the second layer has a fixed retention rate:

$$
\begin{aligned}
& \mathbf{F} p^{i+1}=\mathbf{F} p^{i}+\operatorname{growth} * \operatorname{Convs}\left(\mathbf{F} p^{i}\right), \\
& \operatorname{Convs}(\mathbf{X})=\operatorname{Relu}\left(\operatorname{Bn}\left(\operatorname{Conv}_{1 D}(\mathbf{X})\right)\right),
\end{aligned}
$$

where $\mathbf{F} p^{i+1}$ is the feature map obtained by the $i+1$ th layer, $\mathbf{F} p^{i}$ is the feature map obtained by the $i$ th layer, growth is the retention rate, Relu is the ReLu nonlinear activation function, $B n$ is the batch normalisation layer, and $C o n v_{1 D}$ is the one-dimensional convolution with a kernel size of 15 . By design, multi-scale protein features can be preserved for the final representation of a protein.

\section{The ProbSparse Self-Attention Module}

Most of the existing CPI prediction models simply concatenate the compound and protein features as the input to a classifier generating the CPI prediction. However, such a model may convert the CPI prediction task into a compound or protein classification task. To avoid this problem, it is important to focus on the interaction information between a compound and a protein through a suitable attention mechanism. Unlike computer vision tasks, we cannot obtain the CPI attention information by applying merely channel or spatial attention to the protein feature map because learning attention requires capturing the information on the interaction of the protein and compound features. This requirement is similar to the needs of the machine translation task in natural language processing. However, in contrast to natural language processing where high quality semantic information is available for training, CPI prediction relies on more ambiguous relationships between different data modalities. Hence, an appropriate attention module for CPI prediction reflecting this nuance should carefully be designed.

We would like the attention module not only to improve the prediction accuracy, but also to localise the interaction area. The conventional self-attention mechanism meets this goal to some extent. However, the self-attention mechanism has too many parameters, and the information extracted tends to be redundant. Moreover, the generated attention weight matrix is very complicated, dramatically increasing the difficulty of feature visualisation. Hence, this basic self-attention mechanism for CPI prediction needs to be further refined.

Note that the high-response region of attention contributes more to the prediction results, and we tend to record these regions where interaction occurs. Conversely, a low response often imposes little influence on the prediction, due to the feature transmission process, and it becomes noninformative. Thus, the low attention regions can simultaneously affect both, the computational efficiency of the model and its accuracy. For these reasons, we use the ProbSparse self-attention module of the Informer model to remove the low-attention response area [33]. This helps to optimise the overall model and improve its performance in terms of both efficiency and accuracy.

As shown in Fig. 5, the key idea of Informer is to replace the query matrix in the Transformer model with a sparse matrix $\overline{\mathbf{Q}}$ of the same size of $\mathbf{Q}$ :

$$
\begin{gathered}
A(\mathbf{Q}, \mathbf{K}, \mathbf{V})=\operatorname{Softmax}\left(\frac{\overline{\mathbf{Q}} \mathbf{K}^{T}}{\sqrt{d}}\right) \mathbf{V} . \\
\bar{M}\left(q_{i}, \mathbf{K}\right)=\max \left\{\frac{q_{i} k_{j}^{T}}{\sqrt{d}}\right\}-\frac{1}{L^{K}} \sum_{j=1}^{L^{K}} \frac{q_{i} k_{j}^{T}}{\sqrt{d}} .
\end{gathered}
$$

$\overline{\mathbf{Q}}$ only contains the top-u queries under the sparsity measurement $\bar{M}\left(q_{i}, \mathbf{K}\right)$, the value of $u$ is controlled by a constant sampling factor $c\left(u=c \cdot \ln L_{Q}\right), \ln L_{Q}$ is the number of columns of the matrix $\overline{\mathbf{Q}}, q_{i}$ is the $i$ th column vector of the Query matrix, $k_{j}$ is the $j$ th column vector of the Key matrix, $L^{K}$ is formed by the rows of the Key matrix, and $d$ is the input dimension. For more details of the above two equations, the reader is referred to [33].

\section{Prediction Head and Loss Function}

As shown in Fig. 2, we concatenate the protein and compound feature maps obtained by applying different attention mechanisms for CPI prediction. Specifically, we apply two convolutional layers and three fully connected layers to predict the interaction of a compound and a protein. This is a conventional module that is suitable for a classification task. The size of the convolution kernel is 19, and the length of the fully connected layer is $(75,75,2)$.

For the end-to-end network training, we use the CrossEntropy loss function [42]. Note that we apply the Logsoftmax [43] nonlinear activation layer to the output features before calculating the loss:

$\operatorname{loss}(p, q)=-\sum_{i=1}^{M} \mathbf{P}(i) \log (\mathbf{Q}(i))+(1-\mathbf{P}(i)) \log (1-\mathbf{Q}(i))$,

where $p$ is the true class label, $q$ is the predicted class label, and $M$ is the total number of classes. 
TABLE I

Details of the Benchmarking DATASETs: Human [24], C.elegans [24], BindingDB [30], GPCRs And Kinase [3].

\begin{tabular}{l|rr|rrrr}
\hline Dataset & Partition & Label Reversal & Num. of Proteins & Num. of Compounds & Num. of Positive Pairs & Num. of Negative Pairs \\
\hline Human & None & NO & 852 & 1,052 & 3,369 & 2,843 \\
C.elegans & None & NO & 2,504 & 1,434 & 4,000 & 3,511 \\
BindingDB & Train & NO & 758 & 43,160 & 28,240 & 21,915 \\
& Dev & & 472 & 5,077 & 2,831 & 2,776 \\
& Test & & 466 & 5,016 & 2,706 & 7,269 \\
GPCRs & Train & YES & 243 & 4,359 & 720 & 6,537 \\
& Test & & 113 & 1,000 & 19,200 & 817 \\
Kinases & Train & YES & 153 & 1,044 & 3,990 & 72,352 \\
& Test & & 76 & 600 & 15,695 \\
\hline
\end{tabular}

\section{EXPERIMENTAL RESULTS}

In this section, we first introduce the benchmarking datasets used for the evaluation, and the implementation details. Then we analyse the impact of each innovative component of our CPInformer method in the ablation study. Third, we report the results obtained by our method on all the benchmarks to compare with the state-of-the-art approaches. Last, we demonstrate the capability of the proposed method in visualising compound-protein interactions.

\section{A. The Experimental Settings and Implementation Details}

We evaluate the proposed CPInformer method on five datasets, including Human [24], C.elegans [24], BindingDB [30], GPCRs and Kinase [3]. Our evaluation approach follows [3]. The Humans and C.elegans datasets are evaluated by the 5-fold cross-validation protocol. The BindingDB, GPCRs, and Kinases datasets are usually divided into training, validation, and test sets. However, in some cases the data partition methods on the Humans and C.elegans dataset are unknown, e.g. [28] and [24]. In this paper, we follow the partition method used in [3] for evaluation.

- The Human dataset has 3,369 positive interactions between 1,052 unique compounds and 852 unique proteins.

- The C.elegans dataset contains 4,000 positive interactions between 1,434 unique compounds and 2,504 unique proteins.

- The BindingDB dataset has 33,777 positive samples and 27,003 negative samples. This dataset is divided into training, validation and test subsets. The validation subset is used for hyper-parameter tuning and the results are reported on the test subset.

- The GPCRs dataset has 7,989 positive interactions between 5,359 unique compounds and 356 proteins.

- Kinase has 23,190 positive interactions between 1,644 unique compounds and 229 proteins.

Although the number of the types of the proteins and compounds in the Kinases dataset is small, the total number of samples is large, which leads to a poor generalisation performance of the CPI prediction model. We alleviate this problem to a certain extent by using sample balancing methods and label smoothing. This notably improves the performance of the proposed CPInformer model on this dataset. More details of the above datasets are presented in Table I.
In this paper, we use the Area Under Receiver Operating Characteristic Curve (AUC-ROC) and Area Under PrecisionRecall Curve (AUC-PR) as our main evaluation metrics. To be more specific, the ROC curve plots the true positive rate along the $\mathrm{Y}$-axis and false positive rate on the $\mathrm{X}$-axis. The PR curve plots the precision and recall values on $\mathrm{Y}$-axis and $\mathrm{X}$-axis, respectively. We also use the precision and recall metrics for some experiments by following standard evaluation protocols.

The proposed method was implemented in Python3.7 powered by PyTorch 1.6.0. The experiments were carried out on a machine with Ubuntu 16.04, Intel Xeon Gold 6134 CPU @ 3.20 GHz and one NVIDIA GeForce RTX 2080Ti card. We used the AdamW optimiser for network training. All the hyperparameter settings used in the proposed method are listed in Table III.

\section{B. Ablation Study}

In this part, we first analyse the effectiveness of each innovative element of our proposed CPInformer method. Then we study the impact of different feature extraction settings on the performance of our CPInformer model.

1) Analysis of Different Innovative Elements: To verify the effectiveness of each proposed innovative element of our CPInformer, we first define our baseline method. The baseline model extracts protein features by a three-layer CNN network and compound features by a three-layer GCN network. The baseline model also uses the self-attention mechanism to gather the information about the interaction of proteins and compounds. For the prediction head of the baseline model, we use the same convolutional and fully connected layers as CPInformer to predict CPI interactions. We report the results of our baseline model and the models with different configurations of our innovative components in Table II. The baseline method achieves $0.885,0.935$ and 0.925 in term of AUC-ROC on the Human, C.elegans and BindingDB datasets.

We then tested the effectiveness of the proposed compound feature fusion and multi-scale protein feature extraction modules separately using Model-1 and Model-2. We can see that both models improve the performance of the baseline method on the benchmarking datasets significantly, with the exception of the results obtained by Model-2 on BindingDB. This validates our argument that a richer and discriminative representation of the compounds and proteins is crucial to solving the CPI prediction task successfully. The compound feature fusion module replaces the original GCN features by 
TABLE II

THE ABLATION STUDY RESUlTS OBTAINED ON THREE PUBLIC DATASETS, INCLUDING HUMAN, C.ELEGANS AND BINDINGDB, REPORTING AUC-ROC. CFF: Compound Feature Fusion. MPFE: Multi-scale Protein Feature Fusion. PSSA: ProbSparse Self Attention.

\begin{tabular}{l|rrr|rrr}
\hline Model & CFF & MPFE & PSSA & Human & C.elegans & BindingDB \\
\hline Baseline & & & & 0.885 & 0.935 & 0.925 \\
\hline Model-1 & $\sqrt{ }$ & & & $\uparrow 0.943$ & $\uparrow 0.979$ & $\uparrow 0.940$ \\
Model-2 & & $\sqrt{ }$ & & $\uparrow 0.968$ & $\uparrow 0.976$ & $\Downarrow 0.918$ \\
Model-3 & $\sqrt{ }$ & $\sqrt{ }$ & & $\uparrow 0.973$ & $\uparrow 0.986$ & $\uparrow 0.954$ \\
\hline Model-4 & & & $\sqrt{ }$ & $\Downarrow 0.882$ & $\uparrow 0.939$ & $\uparrow 0.928$ \\
Model-5 & $\sqrt{ }$ & $\sqrt{ }$ & $\sqrt{ }$ & $\uparrow 0.985$ & $\uparrow 0.991$ & $\uparrow 0.965$ \\
\hline
\end{tabular}

TABLE III

HYPER-PARAMETER SETTINGS OF OUR CPINFORMER.

\begin{tabular}{l|r}
\hline Hyper-parameter & Value \\
\hline Learning rate & $5 \times 10^{-4}$ \\
Weight decay & $1 \times 10^{-2}$ \\
The number of GCN layers & 3 \\
The number of CNN layers & 5 \\
The CNN kernel size $k$ & $1 \times 15$ \\
The growth rate of each dense block & 15 \\
The number of attention heads & 5 \\
The value of factor $c$ in Informer & 5 \\
Batch size & 4 \\
Dropout & 0.1 \\
\hline
\end{tabular}

adding the functional group information, which helps to distinguish molecules with similar planar structures. The compound feature fusion module improves the performance on the $\mathrm{Hu}$ man, C.elegans, and BindingDB datasets by $5.8 \%, 4.4 \%$, and $1.5 \%$, respectively. The multi-scale protein feature extraction module improves the performance of the baseline method on Human and C.elegans. However, the performance of Model-2 on the BindingDB dataset is worse than that of the baseline method. This demonstrates that, for a large-scale dataset, simply extracting rich protein features is unlikely to perform well when we only use GCN-based compound features. The GCN features do not contain specific chemical characteristics of a compound, which is required to complement the local protein features for high-performance CPI prediction. To verify this conjecture, we use both, the multi-scale protein features and the fused compound features in Model-3. We can see that, the performance of this model is further improved, beating all the previous models with different configurations. The fusion of the multi-scale protein features with our compound features achieves a further performance boost.

To validate the effectiveness of the ProbSparse self-attention module, we first simply replace the original self-attention module in the baseline method by ProbSparse self-attention, resulting in Model-4. However, this only improves the performance on C.elegans and BindingDB slightly. For the Human dataset, the use of ProbSparse self-attention even degrades the performs of the baseline method. The main reason is that the ProbSparse self-attention module requires rich compound and protein features for the attention operation. When we apply the ProbSparse self-attention to the enhanced compound and protein features, Model-5 improves the performance by $1.2 \%, 0.5 \%$, and $1.1 \%$ on the three datasets, as compared with
Model-3 employimg self-attention.

2) Analysis of Different Feature Extraction Settings: As discussed above, rich compound and protein features are very important for high-performance CPI prediction. To study the impact of different feature extraction methods on the performance of our CPInformer, we compare the effect of different settings for compound and protein feature extraction. We first evaluate the performance of our CPInformer using three different types of compound features, including GCN, FCFPs and the proposed fusion method. Then we study the contribution of different convolutional layers to the protein feature extraction. The results obtained on the Human and BindDB datasets are reported in Fig. 6.

According to Fig. 6(a), the performance of the models using different compound feature extraction methods varies significantly, especially on the BindingDB dataset. The AUC$\mathrm{ROC} / \mathrm{PR}$ result of the feature fusion strategy is $2.8 \% / 2.9 \%$ higher than that of GCN and 2.4\%/2.3\% higher than that of FCFPs. The proposed feature fusion method also improves the performance of GCN and FCFPs used separately on the Human dataset by $0.7 \% / 0.7 \%$ and $0.8 \% / 0.5 \%$ respectively. This again demonstrates the effectiveness of the proposed compound feature fusion module.

Fig. 6(b) shows the results of the proposed CPInformer when using different CNN layers for multi-scale protein feature extraction. We can see that the performance of the models with different configurations varies to a small degree. Our method achieves the best performance when using 5 convolutional layers with dense connections for protein feature extraction. The trends of the results suggest that the CPInformer model may perform better when more than ten layers are used for protein feature extraction. However, by trading off the accuracy for a lower computational complexity using a reduced number of parameters of our CPInformer, we finally choose a CNN module with 5 convolutional layers for the multi-scale protein feature extraction.

To verify the superiority of the proposed feature fusion method, we compare it with the fusion methods used in the existing CPI prediction models. For most of the existing interaction prediction tasks (compound-protein interaction, proteinprotein interaction, drug-drug interaction), the current feature fusion methods mainly include concatenation [44], pairwise inner product [45], [46], cross product [47], and elementwise product [48], [49]. We compare these methods with our proposed fusion strategy on three public data sets. The experimental results are reported in Table IV. In contrast with 


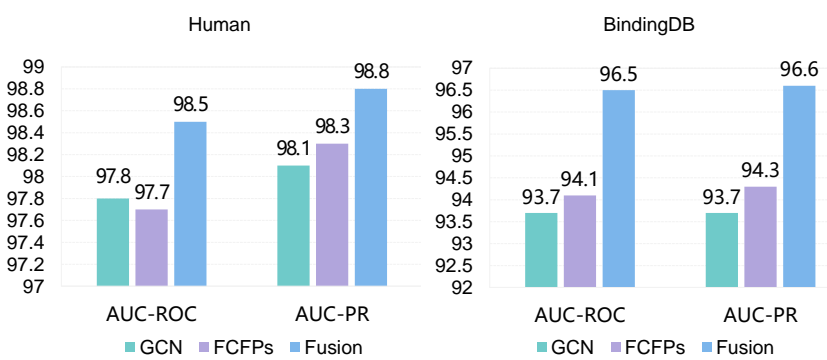

(a) Compound Features
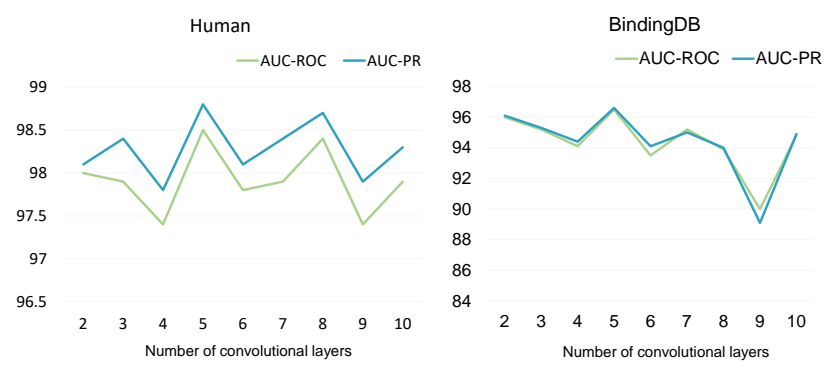

(b) Protein Features

Fig. 6. A comparison of different feature extraction methods for (a) compounds and (b) proteins. For the compound feature extraction, we evaluate three different types of features: GCN, FCFPs and their fusion. For the protein feature extraction, we compare the feature extraction network parameterised with different convolutional layers. The results obtained on the Human and BindingDB datasets are reported in terms of AUC-ROC and AUC-PR.

TABLE IV

A COMPARISON OF THE PROPOSED FEATURE FUSION METHOD WITH THE EXISTING FUSION METHODS. THE RESULTS ARE EVALUATED ON THE HuMAN, C.ELEGANS AND BINDINGDB DATASETS IN TERMS OF AUC-ROC.

\begin{tabular}{l|rrr}
\hline Fusion Methods & Human & C.elegans & BindingDB \\
\hline Concatenation [44] & 0.970 & 0.981 & 0.945 \\
Pairwise inner product [45], [46] & 0.974 & 0.979 & 0.933 \\
Cross product [47] & 0.970 & 0.983 & 0.921 \\
Element-wise product [48], [49] & 0.969 & 0.985 & 0.946 \\
\hline Our proposed & $\mathbf{0 . 9 8 5}$ & $\mathbf{0 . 9 9 1}$ & $\mathbf{0 . 9 6 5}$ \\
\hline
\end{tabular}

the existing methods, the proposed feature fusion method with our attention information mechanism is designed specifically for the proposed CPInformer approach and, as expected, it is more effective.

To further verify the effectiveness of the use of Informer in our method, we compare the proposed method with many other CPI prediction methods, such as DeepconvDTI [20], CPI-GNN [24], TransformerCPI [3], MONN [45] and DeepCDA [50], using different deep learning models. We retain the module used for protein and compound feature extraction in our CPInformer model and use the alternative deep-learningbased CPI prediction modules of the above methods to predict $\mathrm{CPI}$ interactions. As such, we compare the performance of the Informer model with other deep learning models, ensuring that the input features are the same. The results are shown in Table V. The proposed Informer-based method outperforms all the other deep learning approaches, especially on the BindingDB dataset. The MONN and DeepCDA models also achieve excellent performance on the C.elegans dataset. However, their performance degrades significantly on large scale benchmarks such as BindingDB.

\section{Comparison with The State-of-The-Art Methods}

We compare the proposed CPInformer method with the state-of-the-art CPI prediction models, including CPIGNN [24], GraphDTA [25], DeepConvDTI [20] and TransformerCPI [3], on all the five benchmarking datasets. We also compare our method with some traditional approaches, such as K Nearest Neighbours (KNN), Random Forest (RF), L2logistic (L2), Support Vector Machine (SVM) [28] and Graph
TABLE V

A COMPARISON OF INFORMER IN OUR CPINFORMER WITH OTHER DEEP LEARNING MODELS. THE RESULTS, REPORTED IN TERMS OF AUC-ROC, ARE OBTAINED ON THE HUMAN, C.ELEGANS AND BINDINGDB DATASETS.

\begin{tabular}{l|r|rr}
\hline Deep Learning Model & Human & C.elegans & BindingDB \\
\hline CNN (DeepconvDTI [20]) & 0.969 & 0.980 & 0.942 \\
MLP (CPI-GNN [24]) & 0.971 & 0.981 & 0.708 \\
Transformer (TransformerCPI [3]) & 0.973 & 0.986 & 0.954 \\
MONN (MONN [45]) & 0.964 & 0.990 & 0.938 \\
LSTM (DeepCDA [50]) & 0.972 & 0.989 & 0.941 \\
\hline Informer (CPInformer) & $\mathbf{0 . 9 8 5}$ & $\mathbf{0 . 9 9 1}$ & $\mathbf{0 . 9 6 5}$ \\
\hline
\end{tabular}

Convolution Networks (GCN). For these methods, we reuse the results reported in [3].

1) Results on Public Datasets: We first evaluate our CPInformer model on three public datasets, including Human, C.elegans and BindingDB. The Human and C.elegans datasets are relatively small, compared to the other datasets. Additionally, Human and C.elegans do not specifically divide a dataset into training and test sets. For a fair comparison, we follow the setting used in TransformerCPI [3] to evaluate our method on the Human and C.elegans datasets. For BindingDB, we follow the evaluation protocol provided by the dataset.

The results obtained on the Human and C.elegans datasets are reported in Table VI. We use AUC-ROC, AUC-PR, precision and recall as the evaluation metrics. According to the table, the proposed CPInformer achieves a superior performance over all the other methods in terms of AUC-ROC and AUC-PR. For the Human dataset, our CPInformer method achieves the best result in terms of AUC-ROC with 1.2\% performance gain, as compared with the second best method, TransformerCPI. Although the performance of the proposed method is slightly worse than DeepConvDTI [20] in terms of recall, we outperform DeepConvDTI [20] significantly on the main evaluation metrics, AUC-ROC, AUC-PR and precision. Our CPInformer also beats all the other approaches in terms of recall on the C.elegans dataset. Although TransformerCPI [3] performed slightly better than our CPInformer in terms of precision, we outperform TransformerCPI [3] on all the other metrics.

The results obtained on the BindingDB dataset are shown in Table VII. BindingDB is a large-scale dataset as com- 
TABLE VI

A COMPARISON WITH THE STATE-OF-THE-ART METHODS IN TERMS OF AUC-ROC,AUC-PR, PRECISION, AND RECALL, ON THE HUMAN AND C.ELEGANS DATASETS. THE RESUlTS OBTAINED By THE METHODS WITH '*' ARE BASED ON OUR OWN IMPLEMENTATIONS.

\begin{tabular}{|c|c|c|c|c|c|c|c|c|}
\hline \multirow{2}{*}{ Method } & \multicolumn{4}{|c|}{ Human } & \multicolumn{4}{|c|}{ C.elegans } \\
\hline & AUC-ROC & AUC-PR & Precision & Recall & AUC-ROC & AUC-PR & Precision & Recall \\
\hline $\mathrm{KNN}^{*}[28]$ & $0.862 \pm 0.008$ & $0.871 \pm 0.009$ & $0.927 \pm 0.005$ & $0.798 \pm 0.012$ & $0.858 \pm 0.008$ & $0.862 \pm 0.009$ & $0.801 \pm 0.010$ & $0.827 \pm 0.009$ \\
\hline $\mathrm{RF}^{*}[28]$ & $0.940 \pm 0.004$ & $0.951 \pm 0.003$ & $0.897 \pm 0.003$ & $0.861 \pm 0.003$ & $0.902 \pm 0.007$ & $0.896 \pm 0.005$ & $0.821 \pm 0.006$ & $0.844 \pm 0.007$ \\
\hline L2* [28] & $0.911 \pm 0.010$ & $0.921 \pm 0.009$ & $0.913 \pm 0.007$ & $0.867 \pm 0.008$ & $0.892 \pm 0.004$ & $0.903 \pm 0.005$ & $0.890 \pm 0.003$ & $0.877 \pm 0.004$ \\
\hline SVM* [28] & $0.910 \pm 0.023$ & $0.923 \pm 0.026$ & $0.910 \pm 0.023$ & $0.939 \pm 0.025$ & $0.894 \pm 0.003$ & $0.901 \pm 0.003$ & $0.785 \pm 0.002$ & $0.818 \pm 0.005$ \\
\hline GraphDTA [25] & $0.960 \pm 0.005$ & $0.965 \pm 0.004$ & $0.882 \pm 0.040$ & $0.912 \pm 0.040$ & $0.974 \pm 0.004$ & $0.979 \pm 0.003$ & $0.927 \pm 0.015$ & $0.912 \pm 0.023$ \\
\hline GCN [3] & $0.956 \pm 0.004$ & $0.960 \pm 0.003$ & $0.862 \pm 0.006$ & $0.928 \pm 0.010$ & $0.975 \pm 0.004$ & $0.981 \pm 0.007$ & $0.921 \pm 0.008$ & $0.927 \pm 0.006$ \\
\hline CPI-CNN* [24] & $0.970 \pm 0.003$ & $0.973 \pm 0.002$ & $0.918 \pm 0.001$ & $0.923 \pm 0.004$ & $0.978 \pm 0.002$ & $0.979 \pm 0.003$ & $0.938 \pm 0.002$ & $0.929 \pm 0.002$ \\
\hline DeepConvDTI* [20] & $0.970 \pm 0.003$ & $0.978 \pm 0.003$ & $0.942 \pm 0.002$ & $0.953 \pm 0.003$ & $0.979 \pm 0.003$ & $0.981 \pm 0.002$ & $0.947 \pm 0.002$ & $0.950 \pm 0.001$ \\
\hline TransformerCPI [3] & $0.973 \pm 0.002$ & $0.979 \pm 0.002$ & $0.916 \pm 0.006$ & $0.925 \pm 0.006$ & $0.988 \pm 0.002$ & $0.987 \pm 0.001$ & $0.957 \pm 0.006$ & $0.953 \pm 0.005$ \\
\hline CPInformer & $0.985 \pm 0.003$ & $0.989 \pm 0.002$ & $0.961 \pm 0.002$ & $0.951 \pm 0.002$ & $0.991 \pm 0.002$ & $0.992 \pm 0.001$ & $0.956 \pm 0.002$ & $0.976 \pm 0.001$ \\
\hline
\end{tabular}

TABLE VII

A COMPARISON WITH THE STATE-OF-THE-ART METHODS IN TERMS OF AUC-ROC AND AUC-PR ON THE BINDINGDB DATASET. THE RESULTS OBTAINED BY THE METHODS WITH '*' ARE BASED ON OUR OWN IMPLEMENTATION.

\begin{tabular}{l|rrrr}
\hline Method & AUC-ROC & AUC-PR & Precision & Recall \\
\hline KNN $^{*}[28]$ & 0.672 & 0.684 & 0.617 & 0.689 \\
RF* [28] $^{*}$ [28] & 0.602 & 0.696 & 0.629 & 0.714 \\
SVM $^{*}$ [28] & 0.692 & 0.703 & 0.690 & 0.707 \\
GraphDTA [25] & 0.794 & 0.801 & 0.783 & 0.818 \\
GCN [3] & 0.929 & 0.917 & 0.931 & 0.907 \\
CPI-GNN [24] & 0.927 & 0.913 & 0.929 & 0.897 \\
DeepConvDTI [20] & 0.603 & 0.543 & 0.529 & 0.613 \\
TransformerCPI [3] & 0.944 & 0.947 & 0.933 & 0.941 \\
\hline CPInformer & 0.951 & 0.949 & $\mathbf{0 . 9 5 9}$ & 0.937 \\
\hline
\end{tabular}

pared with Human and C.elegans. According to the table, the performance gains achieved with the proposed method are more significant than those obtained on Human and C.elegans. Our CPInformer method outperforms the second best method, TransformerCPI [3], in terms of the AUC-ROC, AUC-PR, and recall metrics.

To assess the efficiency of the proposed method, we compare CPInformer and the state-of-the-art TransformerCPI method in terms of the number of parameters, training time per epoch and testing time per 1,500 samples, on the three public datasets using the same hardware. The results are reported in Table VIII. As the CPI prediction is a screening task for a large number of samples, the computational efficiency of a model is important. We can see that the model size (number of parameters) of CPInformer is much smaller than that of TransformerCPI. This is reflected in the results of the experimental evaluation. CPInformer outperforms TransformerCPI significantly in terms of training/testing time, demonstrating the superiority of our method over the state-of-the-art.

2) Results on Label Reversal Datasets: In addition to the results obtained on the public datasets, we compare the proposed method with the state-of-the-art approaches on more challenging label reversal benchmarks, GPCRs and Kinases. The results are reported in Table IX.

As shown in Table IX, the proposed CPInformer method has a very good performance on the GCPRs dataset with label
TABLE VIII

A COMPARISON OF CPINFORMER AND TRANSFORMERCPI IN TERMS OF THE NUMBER OF MODEL PARAMETERS, TRAINING TIME AND TEST TIME, ON THE HUMAN, C.ELEGANS AND BINDINGDB DATASETS.

\begin{tabular}{|c|c|c|c|}
\hline \multicolumn{2}{|l|}{ Method } & TransformerCPI & CPInformer \\
\hline \multicolumn{2}{|c|}{ \# Parameters } & $18 \mathrm{M}$ & $1.7 \mathrm{M}$ \\
\hline Training & Human & $187 \mathrm{~s}$ & 43s \\
\hline Time & C.elegans & $270 \mathrm{~s}$ & $46 s$ \\
\hline /Epoch & BindingDB & $930 \mathrm{~s}$ & $660 \mathrm{~s}$ \\
\hline \multicolumn{2}{|c|}{ Test Time /1500 samples } & $12.4 \mathrm{~s}$ & $8.2 \mathrm{~s}$ \\
\hline
\end{tabular}

TABLE IX

A COMPARISON WITH THE STATE-OF-THE-ART METHODS ON GCPRS AND KINASES. THE RESULTS OBTAINED BY THE METHODS WITH ' $*$ ' ARE BASED ON OUR OWN IMPLEMENTATIONS. CPINFORMER+ USES THE PROPOSED DATA BALANCING METHOD.

\begin{tabular}{l|rr|rr}
\hline \multirow{2}{*}{ Method } & \multicolumn{2}{|c|}{ GCPRs } & \multicolumn{2}{c}{ Kinases } \\
\cline { 2 - 5 } & AUC-ROC & AUC-PR & AUC-ROC & AUC-PR \\
\hline GraphDTA [25] & 0.825 & 0.817 & 0.434 & 0.171 \\
GCN [3] [24] & 0.820 & 0.809 & 0.447 & 0.186 \\
CPI-GNN [24] & 0.490 & 0.524 & 0.434 & 0.173 \\
DeepConvDTI* [20] & 0.838 & 0.819 & 0.312 & 0.132 \\
TransformerCPI [3] & 0.863 & 0.859 & 0.598 & 0.280 \\
\hline CPInformer & $\mathbf{0 . 8 8 1}$ & $\mathbf{0 . 8 9 1}$ & $\mathbf{0 . 6 2 4}$ & $\mathbf{0 . 3 3 2}$ \\
CPInformer+ & - & - & $\mathbf{0 . 6 9 8}$ & $\mathbf{0 . 3 8 3}$ \\
\hline
\end{tabular}

reversal, achieving the best results in terms of both AUCROC and AUC-PR. Thanks to the ProbSparse self-attention module, our CPInformer can extract the features characterising the interaction of compounds and proteins, promoting the CPI prediction accuracy. As a result, the proposed CPInformer method achieves $1.8 \%$ and $3.2 \%$ performance gains in terms of AUC-ROC and AUC-PR, as compared with the second best method, TransformerCPI. This is a more significant improvement, compared to the results obtained on the previous datasets, demonstrating the merits of our method for CPI prediction in complex scenarios.

For the results obtained on Kinases in Table IX, unfortunately, the performance of all the methods degrades significantly. Although the AUC-ROC of our method is $2.6 \%$ higher than TransformerCPI, it is still unsatisfactory. This is due to two main reasons. First, the number of the types of proteins and compounds in Kinases is small, but the total 
number of samples is large. This leads to a serious overfitting, and consequently the methods generalise poorly on the test set. The second issue is the imbalance of positive and negative samples. This is also a common problem in many other machine learning tasks. To resolve these two problems, we fine-tune our CPInformer model. First, we use compound features as a mask to intercept protein features so that the protein features of each group of compound-protein samples are more prominent. This method alleviates the problem of insufficient protein types. Also, we use sample balancing and label smoothing to address the data imbalance of positive and negative samples in the training set. As shown in the table (CPInformer+), the above strategies are very effective. Compared to the baseline, the performance on the test set, measured in terms of AUC-ROC and AUC-PR, improves by $7.4 \%$ and $5.1 \%$. The proposed method outperforms all the other approaches significantly.

\section{Visualisation of CPI Interactions}

To visualise the local area of proteins and compounds that signifies a compound-protein interaction is crucial to drug discovery. In this paper, thanks to the proposed attention method, we can use the attention weights to assess the importance of different regions in a compound or a protein marking an interaction. This allows us to understand in which protein domain the interaction occurs and which compound functional groups dominates the interaction.

In Fig. 7, we visualise two compound-protein interactions as examples using the Pymol [35] toolbox. More visualisation examples can be found in the Appendix. For visualisation, if both, the compound and the protein sequence exist in the RCSB PDB database [51], we directly download the corresponding PDB file and use Pymol to visualise the protein sequence features based on the attention information obtained by our attention method. If a new ligand is involved, we first use the Rosetta [52] tool to dock the protein and ligand to obtain the PDB file. Then we use Pymol for visualisation. The left hand side of the figure shows the bird-eye view of an interaction, in which the pink section of the protein signifies the area with the highest probability of the interaction. We highlight the local area of a protein with the pink colour where the ProbSparse self-attention mechanism produces high responses. In contrast, the areas with lower attention responses, where the interaction is likely to occur with a lower probability, are rendered in blue. By zooming into the pink area to analyse the interaction in more details, we can see the specific location of the compound that interacts with the protein.

To check how the atoms and functional groups in a compound molecule affect an interaction, we zoom in further and highlight the high-frequency area based on the attention and FCFPs information, resulting in more detailed graphs in Fig. 8. In feature fusion, we can also visualise the functional groups that consolidate compound molecules and proteins to interact according to the weight of the influence of FCFPs in the molecular graph. We randomly visualise the molecular diagrams of 6 compounds in the Human dataset. The coloured areas mark the higher attention weights in the model. These

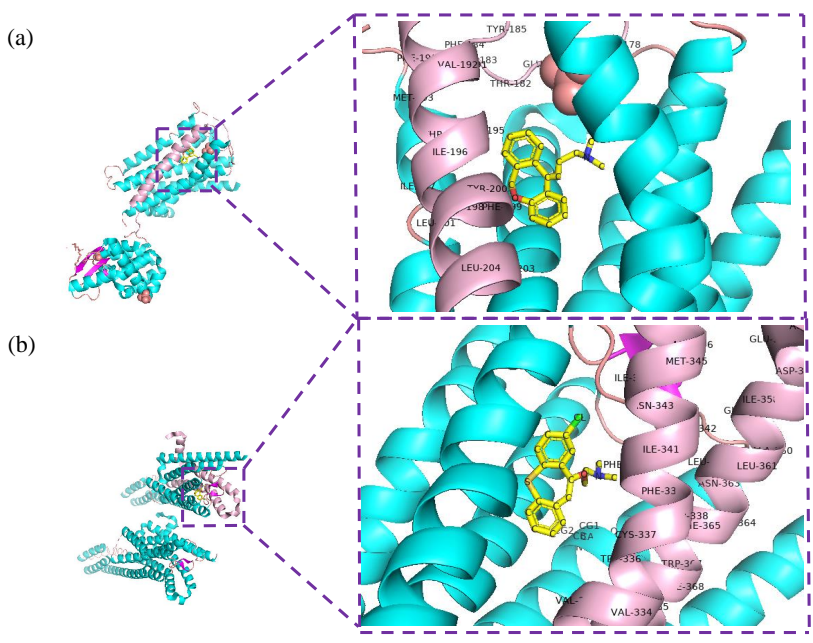

Fig. 7. Visualisation of two compound-protein interactions: (a) the interaction between the Cyproheptadine compound and Histamine $\mathrm{H} 1$ receptor, (b) the interaction between the Cyclobenzaprine compound and the 5hydroxytryptamine receptor $2 \mathrm{~A}$. The pink areas are highlighted by high responses obtained by the ProbSpare self-attention mechanism. We also show the zoom-in images of compounds on the right hand side. They contain the atomic composition of the compound and the name of the amino acid in the region where the protein interacts.
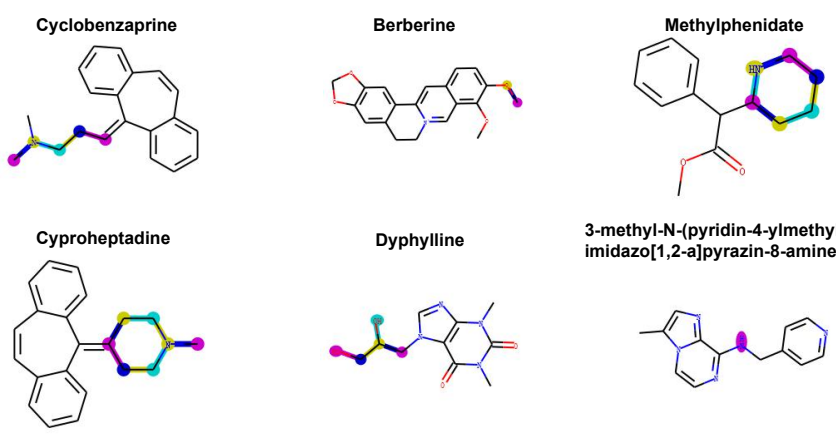

3-methyl-N-(pyridin-4-ylmethyl) imidazo[1,2-a]pyrazin-8-amine

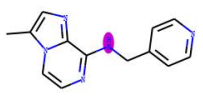

Fig. 8. The weights of the compounds of six positive samples in the Human dataset. The highlighted parts are the areas that have a more critical influence on the CPI interactions. The Drugbank IDs of the compounds and the Uniprot IDs of the proteins of the six samples are: DB00924 \& P28223, DB04115 \& O15392, DB00422 \& Q01959, DB00434 \& P35367, DB00651 \& Q07343, and DB08533 \& P24941.

areas are the positions that the model has inferred after learning from a large amount of data. It can help us to analyse and understand the instances of compound-protein interaction. This aid is very useful for drug discovery and design.

With the visualisation results, it is easy to find the vital functional groups in a compound that determine whether an interaction occurs. It demonstrates that the use of the functional group information is crucial for high-performance CPI prediction, validating the proposed feature fusion method. We also find that the 3D structural information affects the interaction potential enormously. Therefore, adding this information to a CPI prediction model is important for future studies. 


\section{CONCLUSION}

We presented a novel CPInformer model for efficient and robust CPI prediction. The proposed method focused on enhancing the features extracted from compounds and proteins. We fused the FCFPs and structural GCN features for rich compound feature extraction. The proposed approached reduced the prediction error caused by the compounds with similar structures. In addition, we used dense connections to extract multi-scale protein features, preserving local and global information for high-performance CPI prediction. Last, the Informer method with probabilistic sparse self-attention was used to fuse the compound and protein features, reducing information redundancy and enhancing the discriminative power of the fused features for CPI prediction.

The extensive experimental results obtained on five wellknown benchmarking datasets verified the superiority of our method over the state-of-the-art approaches in terms of accuracy and efficiency. Furthermore, we demonstrated that the proposed method is able to visualise the state of a compounddrug interaction, localising the docking site of the protein and compound by means of the attention weights. This allows biologists to screen drugs more conveniently. Our CPInformer model has the potential to facilitate virtual drug screening, but there is still room for improvement. For example, in our future work, we will consider how to fuse multi-level structural features of proteins more effectively and efficiently.

\section{ACKNOWLEDGEMENTS}

This work was supported in part by the National Key Research and Development Program of China (2017YFC1601800), the National Natural Science Foundation of China $(61876072,61902153,62072243)$, the China Postdoctoral Science Foundation (2018T110441) and the Six Talent Peaks Project of Jiangsu Province (XYDXX-012).

\section{REFERENCES}

[1] Peter Kuhn, Keith Wilson, Marianne G Patch, and Raymond C Stevens. The genesis of high-throughput structure-based drug discovery using protein crystallography. Current opinion in chemical biology, 6(5):704 710, 2002.

[2] Eric E Swayze, Elizabeth A Jefferson, Kristin A Sannes-Lowery, Lawrence B Blyn, Lisa M Risen, Satoshi Arakawa, Stephen A Osgood, Steven A Hofstadler, and Richard H Griffey. Sar by ms: a ligand based technique for drug lead discovery against structured rna targets. Journal of medicinal chemistry, 45(18):3816-3819, 2002.

[3] Lifan Chen, Xiaoqin Tan, Dingyan Wang, Feisheng Zhong, Xiaohong Liu, Tianbiao Yang, Xiaomin Luo, Kaixian Chen, Hualiang Jiang, and Mingyue Zheng. Transformercpi: improving compoundprotein interaction prediction by sequence-based deep learning with selfattention mechanism and label reversal experiments. Bioinformatics, 36(16):4406-4414, 2020.

[4] Markus Bredel and Edgar Jacoby. Chemogenomics: an emerging strategy for rapid target and drug discovery. Nature Reviews Genetics, 5(4):262-275, 2004

[5] Kevin Bleakley and Yoshihiro Yamanishi. Supervised prediction of drug-target interactions using bipartite local models. Bioinformatics, 25(18):2397-2403, 2009.

[6] Feixiong Cheng, Yadi Zhou, Jie Li, Weihua Li, Guixia Liu, and Yun Tang. Prediction of chemical-protein interactions: multitarget-qsar versus computational chemogenomic methods. Molecular BioSystems, 8(9):2373-2384, 2012.

[7] Mehmet Gönen. Predicting drug-target interactions from chemical and genomic kernels using bayesian matrix factorization. Bioinformatics, 28(18):2304-2310, 2012.
[8] Laurent Jacob and Jean-Philippe Vert. Protein-ligand interaction prediction: an improved chemogenomics approach. Bioinformatics, 24(19):2149-2156, 2008

[9] Twan van Laarhoven, Sander B Nabuurs, and Elena Marchiori. Gaussian interaction profile kernels for predicting drug-target interaction. Bioinformatics, 27(21):3036-3043, 2011.

[10] Fei Wang, Dongxiang Liu, Heyao Wang, Cheng Luo, Mingyue Zheng, Hong Liu, Weiliang Zhu, Xiaomin Luo, Jian Zhang, and Hualiang Jiang. Computational screening for active compounds targeting protein sequences: methodology and experimental validation. Journal of chemical information and modeling, 51(11):2821-2828, 2011.

[11] Yuhao Wang and Jianyang Zeng. Predicting drug-target interactions using restricted boltzmann machines. Bioinformatics, 29(13):i126-i134, 2013.

[12] Yoshihiro Yamanishi, Michihiro Araki, Alex Gutteridge, Wataru Honda, and Minoru Kanehisa. Prediction of drug-target interaction networks from the integration of chemical and genomic spaces. Bioinformatics, 24(13):i232-i240, 2008

[13] Kai Tian, Mingyu Shao, Yang Wang, Jihong Guan, and Shuigeng Zhou. Boosting compound-protein interaction prediction by deep learning. Methods, 110:64-72, 2016.

[14] Masatoshi Hamanaka, Kei Taneishi, Hiroaki Iwata, Jun Ye, Jianguo Pei, Jinlong Hou, and Yasushi Okuno. Cgbvs-dnn: Prediction of compoundprotein interactions based on deep learning. Molecular informatics, 36(12): 1600045,2017

[15] Fangping Wan and Jianyang Michael Zeng. Deep learning with feature embedding for compound-protein interaction prediction. bioRxiv, page 086033, 2016.

[16] Yoshihiro Yamanishi, Masaaki Kotera, Minoru Kanehisa, and Susumu Goto. Drug-target interaction prediction from chemical, genomic and pharmacological data in an integrated framework. Bioinformatics, 26(12):i246-i254, 2010

[17] Jian-Ping Mei, Chee-Keong Kwoh, Peng Yang, Xiao-Li Li, and Jie Zheng. Drug-target interaction prediction by learning from local information and neighbors. Bioinformatics, 29(2):238-245, 2013.

[18] Salvatore Alaimo, Alfredo Pulvirenti, Rosalba Giugno, and Alfredo Ferro. Drug-target interaction prediction through domain-tuned network-based inference. Bioinformatics, 29(16):2004-2008, 2013.

[19] Ruolan Chen, Xiangrong Liu, Shuting Jin, Jiawei Lin, and Juan Liu. Machine learning for drug-target interaction prediction. Molecules, 23(9):2208, 2018

[20] Ingoo Lee, Jongsoo Keum, and Hojung Nam. Deepconv-dti: Prediction of drug-target interactions via deep learning with convolution on protein sequences. PLoS computational biology, 15(6):e1007129, 2019.

[21] David Rogers and Mathew Hahn. Extended-connectivity fingerprints. Journal of chemical information and modeling, 50(5):742-754, 2010.

[22] Hakime Öztürk, Elif Ozkirimli, and Arzucan Özgür. Widedta: prediction of drug-target binding affinity. arXiv preprint arXiv:1902.04166, 2019.

[23] Steven Kearnes, Kevin McCloskey, Marc Berndl, Vijay Pande, and Patrick Riley. Molecular graph convolutions: moving beyond fingerprints. Journal of computer-aided molecular design, 30(8):595-608, 2016.

[24] Masashi Tsubaki, Kentaro Tomii, and Jun Sese. Compound-protein interaction prediction with end-to-end learning of neural networks for graphs and sequences. Bioinformatics, 35(2):309-318, 2019.

[25] Thin Nguyen, Hang Le, Thomas P Quinn, Thuc Le, and Svetha Venkatesh. Graphdta: Predicting drug-target binding affinity with graph neural networks. biorxiv. April 2020a. doi, 10(684662):684662, 2020.

[26] UniProt Consortium. Uniprot: a hub for protein information. Nucleic acids research, 43(D1):D204-D212, 2015.

[27] Ashish Vaswani, Noam Shazeer, Niki Parmar, Jakob Uszkoreit, Llion Jones, Aidan N Gomez, Lukasz Kaiser, and Illia Polosukhin. Attention is all you need. arXiv preprint arXiv:1706.03762, 2017.

[28] Hui Liu, Jianjiang Sun, Jihong Guan, Jie Zheng, and Shuigeng Zhou. Improving compound-protein interaction prediction by building up highly credible negative samples. Bioinformatics, 31(12):i221-i229, 2015 .

[29] Tiqing Liu, Yuhmei Lin, Xin Wen, Robert N Jorissen, and Michael K Gilson. Bindingdb: a web-accessible database of experimentally determined protein-ligand binding affinities. Nucleic acids research, 35(suppl_1):D198-D201, 2007.

[30] Kyle Yingkai Gao, Achille Fokoue, Heng Luo, Arun Iyengar, Sanjoy Dey, and Ping Zhang. Interpretable drug target prediction using deep neural representation. In IJCAI, volume 2018, pages 3371-3377, 2018.

[31] Sereina Riniker and Gregory A Landrum. Similarity maps-a visualization strategy for molecular fingerprints and machine-learning methods. Journal of cheminformatics, 5(1):1-7, 2013 
[32] Gao Huang, Zhuang Liu, Laurens Van Der Maaten, and Kilian Q Weinberger. Densely connected convolutional networks. In Proceedings of the IEEE conference on computer vision and pattern recognition, pages $4700-4708,2017$

[33] Haoyi Zhou, Shanghang Zhang, Jieqi Peng, Shuai Zhang, Jianxin Li, Hui Xiong, and Wancai Zhang. Informer: Beyond efficient transformer for long sequence time-series forecasting. arXiv preprint arXiv:2012.07436, 2020.

[34] Lei Deng and Zhigang Chen. An integrated framework for functional annotation of protein structural domains. IEEE/ACM transactions on computational biology and bioinformatics, 12(4):902-913, 2015.

[35] Warren L DeLano et al. Pymol: An open-source molecular graphics tool. CCP4 Newsletter on protein crystallography, 40(1):82-92, 2002.

[36] A Patrícia Bento, Anne Hersey, Eloy Félix, Greg Landrum, Anna Gaulton, Francis Atkinson, Louisa J Bellis, Marleen De Veij, and Andrew R Leach. An open source chemical structure curation pipeline using rdkit. Journal of Cheminformatics, 12(1):1-16, 2020.

[37] Franco Scarselli, Marco Gori, Ah Chung Tsoi, Markus Hagenbuchner, and Gabriele Monfardini. The graph neural network model. IEEE transactions on neural networks, 20(1):61-80, 2008

[38] Hexiang Deng, Christian J Doonan, Hiroyasu Furukawa, Ricardo B Ferreira, John Towne, Carolyn B Knobler, Bo Wang, and Omar M Yaghi. Multiple functional groups of varying ratios in metal-organic frameworks. Science, 327(5967):846-850, 2010.

[39] James C Whisstock and Arthur M Lesk. Prediction of protein function from protein sequence and structure. Quarterly reviews of biophysics, $36(3): 307,2003$

[40] K Wüthrich, M Billeter, and W Braun. Pseudo-structures for the 20 common amino acids for use in studies of protein conformations by measurements of intramolecular proton-proton distance constraints with nuclear magnetic resonance. Journal of molecular biology, 169(4):949961,1983

[41] Chao Che, Min Zhu, Yongjun Zhu, Qiang Zhang, Dongsheng Zhou, and Bin Wang. A protein embedding model for drug molecular screening. In 2020 IEEE International Conference on Big Data and Smart Computing (BigComp), pages 251-254. IEEE, 2020.

[42] Zhilu Zhang and Mert R Sabuncu. Generalized cross entropy loss for training deep neural networks with noisy labels. arXiv preprint arXiv:1805.07836, 2018.

[43] Alexandre de Brébisson and Pascal Vincent. An exploration of softmax alternatives belonging to the spherical loss family. arXiv preprint arXiv:1511.05042, 2015.

[44] Ping Xuan, Yu Zhang, Hui Cui, Tiangang Zhang, Maozu Guo, and Toshiya Nakaguchi. Integrating multi-scale neighbouring topologies and cross-modal similarities for drug-protein interaction prediction. Briefings in Bioinformatics, 22(5):bbab119, 2021.

[45] Shuya Li, Fangping Wan, Hantao Shu, Tao Jiang, Dan Zhao, and Jianyang Zeng. Monn: a multi-objective neural network for predicting compound-protein interactions and affinities. Cell Systems, 10(4):308$322,2020$.

[46] Zaynab Mousavian and Ali Masoudi-Nejad. Drug-target interaction prediction via chemogenomic space: learning-based methods. Expert opinion on drug metabolism \& toxicology, 10(9):1273-1287, 2014.

[47] Selma Dere and Serkan Ayvaz. Prediction of drug-drug interactions by using profile fingerprint vectors and protein similarities. Healthcare informatics research, 26(1):42-49, 2020.

[48] Narumi Watanabe, Yuuto Ohnuki, and Yasubumi Sakakibara. Deep learning integration of molecular and interactome data for proteincompound interaction prediction. Journal of Cheminformatics, 13(1):112, 2021.

[49] Yujie Chen, Tengfei Ma, Xixi Yang, Jianmin Wang, Bosheng Song, and Xiangxiang Zeng. Muffin: multi-scale feature fusion for drug-drug interaction prediction. Bioinformatics, 2021.

[50] Karim Abbasi, Parvin Razzaghi, Antti Poso, Massoud Amanlou, Jahan B Ghasemi, and Ali Masoudi-Nejad. Deepcda: deep cross-domain compound-protein affinity prediction through $1 \mathrm{stm}$ and convolutional neural networks. Bioinformatics, 36(17):4633-4642, 2020.

[51] Peter W Rose, Bojan Beran, Chunxiao Bi, Wolfgang F Bluhm, Dimitris Dimitropoulos, David S Goodsell, Andreas Prlić, Martha Quesada, Gregory B Quinn, John D Westbrook, et al. The rcsb protein data bank: redesigned web site and web services. Nucleic acids research, 39(suppl_1):D392-D401, 2010.

[52] Ian W Davis and David Baker. Rosettaligand docking with full ligand and receptor flexibility. Journal of molecular biology, 385(2):381-392, 2009.

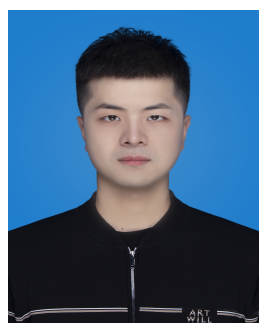

Yang Hua is a $\mathrm{PhD}$ student at the School of Artificial Intelligence and Computer Science, Jiangnan University, Wuxi, China. His research interests include bioinformatics, drug discovery and data mining.

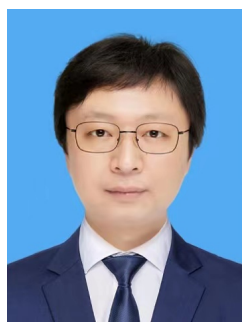

Xiaoning Song received the BSc degree in computer science from Southeast University, Nanjing, China, in 1997, the MSc degree in computer science from the Jiangsu University of Science and Technology, Zhenjiang, China, in 2005, and the $\mathrm{PhD}$ degree in pattern recognition and intelligence system from the Nanjing University of Science and Technology, Nanjing, in 2010. He was a visiting researcher with the Centre for Vision, Speech, and Signal Processing, University of Surrey, Guildford, UK, from 2014 to 2015. He is currently a full Professor with the School of Artificial Intelligence and Computer Science, Jiangnan University, Wuxi, China. His research interests include pattern recognition, machine learning, and computer vision.

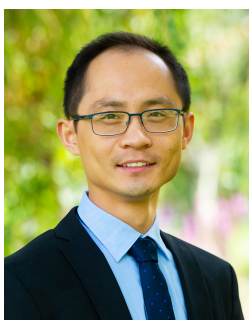

Zhenhua Feng (S'13-M'16) received the Ph.D. degree from the Centre for Vision, Speech and Signal Processing (CVSSP), University of Surrey, Guildford, U.K. in 2016. He is currently a Lecturer in Computer Vision and Machine Learning at the Department of Computer Science, University of Surrey. Before this, he was a senior research fellow at CVSSP. His research interests include pattern recognition, machine learning and computer vision. $\mathrm{He}$ has published more than 60 research papers in top-tier conferences and journals such as TPAMI, IJCV, CVPR, ICCV, IJCAI, ACL, TCYB, TIP, TIFS, TCSVT, TBIOM, ACM TOMM, Information Sciences, Pattern Recognition, etc.

He served as Area Chair for BMVC 2021 and Senior Programme Committee member for IJCAI 2021. He currently serves on the Editorial Board of Complex and Intelligent Systems. He received the 2017 European Biometrics Industry Award from the European Association for Biometrics (EAB) and the Best Paper Award for Commercial Applications from the AMDO2018 conference.

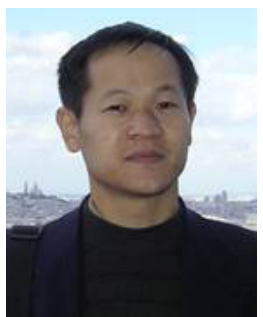

Xiaojun Wu received the BSc degree in mathematics from Nanjing Normal University, Nanjing, China, in 1991, and the M.S. and Ph.D. degrees in pattern recognition and intelligent systems from the Nanjing University of Science and Technology, Nanjing, in 1996 and 2002, respectively. He is currently a Distinguished Professor of Artificial Intelligence and Pattern Recognition with Jiangnan University, Wuxi, China. His current research interests include pattern recognition, computer vision, fuzzy systems, neural networks, and intelligent systems. 


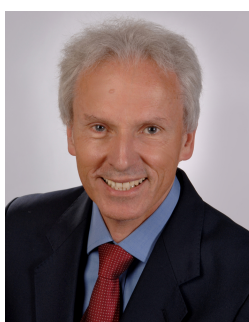

Josef Kittler (M'74-LM'12) received the B.A., $\mathrm{Ph} . \mathrm{D}$., and D.Sc. degrees from the University of Cambridge, in 1971, 1974, and 1991, respectively. $\mathrm{He}$ is a distinguished Professor of Machine Intelligence at the Centre for Vision, Speech and Signal Processing, University of Surrey, Guildford, U.K. He conducts research in biometrics, video and image database retrieval, medical image analysis, and cognitive vision. He published the textbook Pattern Recognition: A Statistical Approach and about 1000 by around 70,000 times. scientific papers. His publications have been cited

$\mathrm{He}$ is series editor of Springer Lecture Notes on Computer Science. He currently serves on the Editorial Boards of Pattern Recognition Letters, Pattern Recognition and Artificial Intelligence, Pattern Analysis and Applications. $\mathrm{He}$ also served as a member of the Editorial Board of IEEE Transactions on Pattern Analysis and Machine Intelligence during 1982-1985. He served on the Governing Board of the International Association for Pattern Recognition (IAPR) as one of the two British representatives during 1982-2005, and the President of IAPR during 1994-1996.

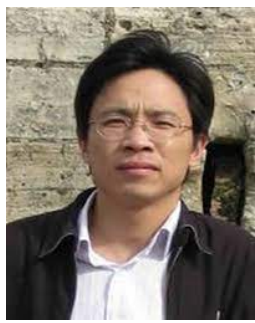

Dongjun Yu received the B.S. degree in computer science and MSc degree in artificial intelligence from the Jiangsu University of Science and Technology, Zhenjiang, China, in 1997 and 2000, respectively, and the Ph.D. degree in pattern analysis and machine intelligence from the Nanjing University of Science and Technology, Nanjing, China, in 2003. In 2008, he was an Academic Visitor at the University of York, U.K. He is currently a Professor at the School of Computer Science and Technology, Nanjing University of Science and Technology, Nanjing, China. His research interests include pattern recognition, data mining, and bioinformatics. 